\title{
Clinicopathological significance of calreticulin in breast invasive ductal carcinoma
}

\author{
Zin-Mar Lwin ${ }^{1}$, Chunhua Guo ${ }^{1}$, Agus Salim², George Wai-Cheong Yip ${ }^{1}$, Fook-Tim Chew ${ }^{3}$, \\ Jiang $\mathrm{Nan}^{3}$, Aye Aye Thike ${ }^{4}$, Puay-Hoon Tan ${ }^{4}$ and Boon-Huat Bay ${ }^{1}$ \\ ${ }^{1}$ Department of Anatomy, Yong Loo Lin School of Medicine, National University of Singapore, Singapore, \\ Singapore; ${ }^{2}$ Department of Epidemiology and Public Health, Yong Loo Lin School of Medicine, National \\ University of Singapore, Singapore, Singapore; ${ }^{3}$ Department of Biological Sciences, National University \\ of Singapore, Singapore, Singapore and ${ }^{4}$ Department of Pathology, Singapore General Hospital, Singapore,
} Singapore

Calreticulin is a chaperone protein located in the lumen of the endoplasmic reticulum. The association of calreticulin with pathological conditions such as autoimmune disorders and certain types of cancer have been reported. However, little is known about its role in the pathogenesis of breast cancer. The aim of this study was to determine the expression of calreticulin in vitro and correlate its expression levels in breast cancer tissue samples with clinicopathological parameters. Calreticulin expression was evaluated in MCF-7 and MDA-MB-231 breast cancer cells by real-time RT-PCR, Western blot, immunohistochemistry, and immunofluorescence staining. Patient tissue microarrays were constructed from 228 breast cancer specimens for immunohistochemical analysis. The in vitro study showed a higher calreticulin expression in more aggressive MDA-MB-231 cells as compared with MCF-7 cells at both mRNA and protein levels. In all, 227 out of 228 breast cancer samples exhibited calreticulin staining in at least $5 \%$ of the cancer cells. Calreticulin immunostaining was observed to be localized to the cytoplasm of the cancer cells. Regression analysis of calreticulin immunostaining in the tissue microarrays revealed that its expression was positively correlated to logarithm of (log) tumor size $(P=0.046)$ and development of distant metastasis $(P=0.017)$. Multivariate analysis confirmed calreticulin expression as an independent predictor of log tumor size and occurrence of distant metastasis. The data suggest that calreticulin expression is associated with more advanced tumors and is a potential prognostic biomarker.

Modern Pathology (2010) 23, 1559-1566; doi:10.1038/modpathol.2010.173; published online 10 September 2010

Keywords: calreticulin; endoplasmic reticulum; immunohistochemistry; invasive ductal breast carcinoma; tissue microarray

Breast cancer is the most frequently occurring cancer among females in many parts of the world. ${ }^{1-3}$ The Surveillance Epidemiology and End Results data estimated that 192370 new cases of breast cancer will be diagnosed in 2009, of which 40170 women will die of the disease.$^{4}$ According to a Singapore Cancer Registry Interim Report released

Correspondence: Dr P-H Tan, MD, FRCPA, Department of Pathology, Singapore General Hospital, Outram Road, Singapore 169608, Singapore or

Dr B-H Bay, MBBS, PhD, Department of Anatomy, Yong Loo Lin School of Medicine, National University of Singapore, 4 Medical Drive, MD10, Singapore 117597, Singapore.

E-mail: tan.puay.hoon@sgh.com.sg or antbaybh@nus.edu.sg

Received 3 May 2010; revised 13 July 2010; accepted 14 July 2010; published online 10 September 2010 in 2009, breast cancer that makes up $29.3 \%$ of all female cancers in Singapore between 2003 and 2007, has taken over the place of lung cancer as the most lethal cancer among females. ${ }^{1}$

Breast cancer is a heterogeneous disease. Although non-invasive cancer includes both ductal and lobular carcinoma in situ, invasive disease has morphological variety, with subtypes incorporating invasive ductal carcinoma, invasive lobular carcinoma, and medullary carcinoma. ${ }^{5}$ Despite advances in early detection techniques, about $80 \%$ of all diagnosed cases are invasive, with the majority accounted for by invasive ductal carcinoma in which malignant cells have already breached the basement membranes to permeate surrounding breast tissues. ${ }^{6}$ Unlike ductal carcinoma in situ, 
which is still confined within its basement membrane, invasive ductal carcinoma has the potential to spread to lymph and blood systems and to distant sites.

The heterogeneity of breast cancer translates to potentially variable treatment responses. It has been observed that breast cancer metastagenicity (likelihood of developing distant metastasis) and virulence are diverse even within clinically related groups of patients afflicted with the disease, ${ }^{7}$ suggesting that there exist molecular characteristics in tumor cells that may make their behavior different from one another. There is also a suggestion that breast cancer is a distinct molecular group of neoplastic disorders. ${ }^{8}$ Some currently established molecular markers of breast cancer include human epidermal growth factor receptor 2 (HER2), estrogen receptor (ER), progesterone receptor (PR), p53, and proliferating cell nuclear antigen. ${ }^{9-14}$ However, even with advances in molecular characterization, $30 \%$ of patients diagnosed with early stage breast cancer developed metastasis. ${ }^{15}$ Hence, there is a continual search for novel and emerging biomarkers as prognosticators of the disease.

Calreticulin is a $46-\mathrm{kDa}$ calcium-binding chaperone protein, which is localized in the lumen of the endoplasmic reticulum. ${ }^{16,17}$ First isolated by Ostwald and MacLennan in $1974,{ }^{18}$ calreticulin is present in a wide range of vertebrates and higher plants. Calreticulin has two main functions, viz., a molecular chaperone and a regulator of intracellular calcium ion homeostasis. ${ }^{19-21}$ Chaperones are a group of proteins that control the proper folding of secreted proteins and membrane-bound surface molecules that are important in communication of the cell with its external environment. As calcium ion is the secondary messenger in many intracellular signaling pathways, calreticulin has an essential role in cell signaling, and deficiency of this protein has been reported to induce embryonic lethality in mice due to failure in cardiac development. ${ }^{22}$

Hence, the aim of this study was to evaluate the expression of calreticulin in breast cancer cells in vitro and tissue samples, and correlate calreticulin immunohistochemical staining with clinicopathological parameters of invasive ductal breast cancer patients, thus exploring the potential of calreticulin as a novel prognostic biomarker for breast cancer.

\section{Materials and methods}

\section{Cell Culture}

Three breast epithelial cell lines used in this study, namely MCF-7, MDA-MB-231, and MCF-12A cell lines, were purchased from American Type Culture Collection. The MCF-7 cell line, which is a noninvasive breast cancer cell line, was routinely maintained in Dulbecco's Modified Eagle's Medium (DMEM) (Sigma) supplemented with 10\% fetal bovine serum (FBS) (Hyclone). MDA-MB-231 cell line, an invasive breast cancer cell line, was maintained in RPMI-1640 (GIBCO) supplemented with $10 \%$ FBS. MCF-12A cells were derived from non-tumorigenic epithelial cells of human mammary gland, and was cultured in DMEM-12F medium (Sigma) supplemented with 5\% FBS, $20 \mathrm{ng} /$ $\mathrm{ml}$ epidermal growth factor, $100 \mathrm{ng} / \mathrm{ml}$ cholera toxin, $0.01 \mathrm{mg} / \mathrm{ml}$ insulin, and $500 \mathrm{ng} / \mathrm{ml}$ hydrocortisone. All cell lines were grown in the presence of $5 \% \mathrm{CO}_{2}$ in a humidified incubator at $37^{\circ} \mathrm{C}$.

\section{Clinical Materials}

Tissue microarrays were constructed as $1 \mathrm{~mm}$ cores (with two cores per case) from paraffin-embedded samples obtained from 228 patients diagnosed with breast invasive ductal carcinoma at the Department of Pathology, Singapore General Hospital, between 2000 and 2006. This study was approved by the Institutional Review Board, Singapore General Hospital. The patients' age ranged from 23 to 89, with mean age of 55.6 years. The tumor size varied from 5 to $140 \mathrm{~mm}$, with a mean size of $34.9 \mathrm{~mm}$. In all, 112 patients had positive lymph node metastasis but none had distant metastasis when first diagnosed with breast cancer. Patients were followed up until June 2009 and during this follow-up period, 17 patients had local recurrence, whereas 18 patients developed distant metastasis. Nine patients had metastasis to the lungs and two patients had distant spread to the liver. The metastatic sites of the other seven patients were the spine, T10 vertebra, T12 vertebra, hip bone, right shoulder soft tissue, left kidney, cervical lymph node and brain. The detailed clinicopathological features of the study population are given in Table 1.

\section{Real-Time RT-PCR}

Total mRNA was extracted using RNeasy Mini kit (Qiagen). After quantification of mRNA concentration by measuring the absorbance at $260 \mathrm{~nm}$ with Bio-Rad spectrophotometer, $1 \mu \mathrm{g}$ was used for cDNA synthesis using Superscript III 1st Strand Synthesis System (Invitrogen) with random hexamer primers following the manufacturer's protocol. The cDNAs synthesized were then subjected to real-time PCR using SYBR-Green (Qiagen) and Roche Real-time LightCycler 2.0 System. The reaction conditions used were one cycle of $95^{\circ} \mathrm{C}$ for $15 \mathrm{~min}$ for activation of Taq DNA polymerase, 40 cycles of $94^{\circ} \mathrm{C}$ for $30 \mathrm{~s}$, $62^{\circ} \mathrm{C}$ for $30 \mathrm{~s}$, and $72^{\circ} \mathrm{C}$ for $60 \mathrm{~s}^{23}$ The primer pairs used for calreticulin were: forward AAG-TTCTAC-GGT-GAC-GAG-GAG, reverse GTC-GAT-GTTCTG-CTC-ATG-TTT-C and for GAPDH were: forward GAA-GGT-GAA-GGT-CGG-AGT-CAA-CG, reverse TGC-CAT-GGG-TGG-AAT-CAT-ATT-GG. The expression level of calreticulin gene was normalized with housekeeping GAPDH gene. The relative expression 
Table 1 Clinicopathological features of the study population $(n=228)$

\begin{tabular}{|c|c|c|}
\hline & $\mathrm{n}$ & $\%$ \\
\hline \multicolumn{3}{|l|}{ Age } \\
\hline$\leq 55$ years & 119 & 52.2 \\
\hline$>55$ years & 106 & 46.5 \\
\hline Unavailable & 3 & 1.3 \\
\hline \multicolumn{3}{|l|}{ Race } \\
\hline Chinese & 185 & 81.1 \\
\hline Malay & 18 & 7.9 \\
\hline Indians & 7 & 3.1 \\
\hline Others & 15 & 6.6 \\
\hline Unavailable & 3 & 1.3 \\
\hline \multicolumn{3}{|l|}{ Tumor size } \\
\hline$\leq 20 \mathrm{~mm}$ & 60 & 26.3 \\
\hline $20 \mathrm{~mm}<\mathrm{T} \leq 50 \mathrm{~mm}$ & 128 & 56.1 \\
\hline$>50 \mathrm{~mm}$ & 30 & 13.2 \\
\hline Unavailable & 10 & 4.4 \\
\hline \multicolumn{3}{|l|}{ AJCC staging } \\
\hline I & 40 & 17.5 \\
\hline IIA/IIB & 106 & 46.5 \\
\hline IIIA/IIIC & 60 & 26.3 \\
\hline Unavailable & 22 & 9.7 \\
\hline \multicolumn{3}{|l|}{ Histological grade ${ }^{\mathrm{a}}$} \\
\hline 1 & 22 & 9.6 \\
\hline 2 & 77 & 33.8 \\
\hline 3 & 126 & 55.3 \\
\hline Unavailable & 3 & 1.3 \\
\hline \multicolumn{3}{|c|}{ Combined histologic score } \\
\hline$\leq 5$ & 15 & 6.6 \\
\hline $6-7$ & 38 & 16.7 \\
\hline$>7$ & 48 & 21.0 \\
\hline Unavailable & 127 & 55.7 \\
\hline \multicolumn{3}{|l|}{ PR expression } \\
\hline Positive & 112 & 49.1 \\
\hline Negative & 115 & 50.4 \\
\hline Unavailable & 1 & 0.5 \\
\hline \multicolumn{3}{|l|}{ ER expression } \\
\hline Positive & 138 & 60.5 \\
\hline Negative & 89 & 39.0 \\
\hline Unavailable & 1 & 0.5 \\
\hline \multicolumn{3}{|l|}{ HER2 expression } \\
\hline Positive & 57 & 25.0 \\
\hline Negative $^{\mathrm{b}}$ & 168 & 73.7 \\
\hline Unavailable & 3 & 1.3 \\
\hline \multicolumn{3}{|c|}{ Lymph node involvement } \\
\hline Yes & 112 & 49.1 \\
\hline No & 99 & 43.4 \\
\hline Unavailable & 17 & 7.5 \\
\hline \multicolumn{3}{|l|}{ Recurrence/metastasis ${ }^{\mathrm{c}}$} \\
\hline Local recurrence & 17 & 7.5 \\
\hline Metastasis & 18 & 7.9 \\
\hline None & 193 & 84.6 \\
\hline
\end{tabular}

${ }^{\mathrm{a}}$ Derived from composite score based on tubule formation, mitotic activity, and nuclear pleomorphism.

${ }^{\mathrm{b}}$ One negative HER2 case was further verified by fluorescence in situ hybridization (FISH).

${ }^{\mathrm{C}}$ Local recurrence or distant metastasis during the follow-up period until June 2009. of the transcript was calculated by $2^{-\Delta \Delta C T}$ using the MCF-12A cell line as the baseline.

\section{Western Blotting}

Total proteins were extracted via direct lysis using M-PER Mammalian Protein Extraction Reagent (Pierce) containing protease inhibitor and EDTA. After quantification, $20 \mu \mathrm{g}$ of protein from each sample was loaded to each well of SDS-acrylamide gel for electrophoresis. The resolved proteins from the gel were then transferred to polyvinylidene fluoride membrane using Bio-Rad TransBlot SD Semi-Dry Transfer Cell unit. The membrane was pre-treated with $5 \%$ non-fat milk over night at $4{ }^{\circ} \mathrm{C}$ before incubating with anti-calreticulin antibody (1:100 dilution; Santa Cruz Biotechnology) at room temperature for $1 \mathrm{~h}$. Horseradish peroxidase conjugated goat anti-rabbit secondary antibody (Sigma) was used at 1:6000 dilution at room temperature for $1 \mathrm{~h}$. The antibody-bound protein band was detected by enhanced chemiluminescense and visualized on an X-ray film. Quantification of protein expression was done by measuring the optical density (OD) of the band on the film using a densitometer (Bio-Rad). The OD ratio was calculated using $\beta$-actin as the internal control and protein sample from MCF-12A cell line as the standard baseline.

\section{Immunohistochemistry}

Immunohistochemical staining was carried out in both MCF-7 and MDA-MB-231 breast cancer cells as well as on patient tissue samples to determine the expression levels and cellular and tissue localization of calreticulin. Commercially available rabbit polyclonal antibody for calreticulin was purchased from Santa Cruz Biotechnology. For each set of immunostaining, a negative control was included to ensure that there is no non-specific staining of the secondary antibody. For the in vitro study, cells were grown separately in Lab-Tek four-chambered coverglass until they reach $80 \%$ confluence before staining. The culture medium was removed from each well, and the cells were washed with phosphate-buffered saline (PBS) at $\mathrm{pH} 7.4$ followed by fixing with $4 \%$ paraformaldehyde for $8 \mathrm{~min}$. The cells were then immunostained manually. Briefly, permeabilization was done by washing the cells a few times with $0.2 \%$ Triton-X in PBS. To eliminate endogenous peroxidase activity, $0.5 \% \quad \mathrm{H}_{2} \mathrm{O}_{2}$ in methanol was added into the culture and incubated at room temperature for $30 \mathrm{~min}$. The cells were pretreated with $5 \%$ goat serum before incubation with 1:100 diluted calreticulin antibody overnight at $4^{\circ} \mathrm{C}$. To detect the primary antibody, biotinylated antirabbit secondary antibody (1:200) was added for $1 \mathrm{~h}$ followed by avidin-biotin peroxidase complex (Vectorstain) for $1 \mathrm{~h}$ at room temperature. Visualization was done with diaminobenzidine (DAB) 
solution for calreticulin, and the nuclei were counterstained with Shandon's hematoxylin. Paraffin-embedded tissue microarray slides were stained using the Bond ${ }^{\mathrm{TM}}$ Automated Immunohistochemistry System by Leica Microsystems. The anti-calreticulin antibody was used at 1:1000 dilution. Dewaxing reagent, antigen retrieval solution, secondary antibody, DAB, and hematoxylin were all provided in the Bond Polymer Detection System.

Results of ER, PR, and HER2 were based on immunohistochemical staining using antibodies to ER (Neomarker RM9101-S at 1:50 dilution), PR (Neomarker RM9102-S at 1:200 dilution) and HER2 (Neomarker RM9103-S at 1:200 dilution). For pretreatment, sections were microwaved at $98^{\circ} \mathrm{C}$ for 12 min in Ventana CCI solution (Ventana Medical Systems). The detection system used was Linked Streptavidin Biotin. For the purpose of this study, a result was considered to be positive for ER and PR if at least $10 \%$ of tumor cells displayed a minimal $2+$ nuclear staining. Although current recommendations for positive hormone receptor status is usually set at $\geq 1 \%$ of reactive tumor cells, we have adopted a previously validated threshold based on correlation with H-scores. ${ }^{24}$ In the case of HER2, a positive test was considered if at least $30 \%$ of tumor cells exhibited $3+$ cell membrane staining. If the above criteria were not fulfilled, the results would be considered as negative.

\section{Quantification of Immunohistochemical Staining}

The stained tissue microarray slides were viewed under a light microscope at $\times 100$ and $\times 400$ to determine the percentage of positively calreticulinstained malignant epithelial cells. Benign epithelium and stromal regions were not included for scoring. The scores were verified independently by a pathologist. Immunoreactivity was represented by weighted average intensity score, which was calculated as immunoreactive score divided by total percentage stained. Immunoreactive score is the sum of the percentage of cells $x$ intensity for each category of weakly stained, moderately stained, and strongly stained cells.

\section{Immunofluorescence Staining}

The procedure for immunofluorescence staining was similar to immunohistochemical staining except that the secondary antibody used was conjugated to fluorochrome TRITC and the nuclei were counterstained with DAPI. The samples were visualized using a laser scanning confocal microscope (Leica).

\section{Statistical Analysis}

For in vitro experiments, namely real-time RT-PCR and western blot results, Student's $t$-test was used to compare the means of calreticulin expression level in MCF-7 cells and MDA-MB-231 cells. For the analysis of patients' clinicopathological data in relation to calreticulin staining, both univariate and multivariate analysis were carried out using STATA version 10 (STATA, College Station, TX, USA). Tumor size, disease staging, histological grade of tumor, combined histological score (based on sum of individual scores of the morphologic parameters of tubule formation, mitotic rate, and nuclear pleomorphism), lymph node involvement, and distant metastasis were identified as outcome variables of interest, whereas patients' age, race, ER, PR, and HER2 expression were identified as potential confounders and controlled for in the analysis. Linear regression was used to model association between log tumor size and weighted average intensity score for calreticulin expression. For other outcome variables, binary logistic regression was used. To enable the use of binary logistic regression, disease staging was regrouped into two categories (early $=$ stage 1 , late $=$ stages 2 and 3 ), histological grade was also regrouped into two categories (lowgrades 1 and 2, high=grade 3 ) and combined histological score into two categories (low and medium $=0-7$, high $=>7$ ). For each of the main outcome of interest, initially the WAI score and potential confounders were included in the model. Then backward stepwise regression was performed in which the non-significant potential confounder with the largest $P$-value of $>0.05$ was dropped from the model. This step was repeated until all confounders retained achieved statistical significance or no confounder was left in the model.

\section{Results}

Calreticulin expression at the mRNA level was found to be significantly higher in MDA-MB-231 cells than in MCF-7 cells $(P<0.001)$ (Figure 1a). The specificity of the primers was confirmed by performing melting curve analysis, which showed a single peak for each primer pair, as well as by $2 \%$ agarose gel electrophoresis, which showed a single band for each pair of primers (not shown). Semiquantitative Western blot analysis further confirmed that MDA-MB-231 has a significantly higher calreticulin expression at protein level when compared with less invasive MCF-7 cells $(P<0.01)$ (Figures $1 \mathrm{~b}$ and c). Calreticulin was found to be localized in the cytoplasm of MCF-7 and MDA-MB231 cells especially in the perinuclear region by immunohistochemical staining (Figure 1d), which concurs with the known fact that calreticulin is an endoplasmic reticulum luminal protein. ${ }^{25}$ The localization and expression of calreticulin in MCF-7 and MDA-MB 231 cancer cells were further verified by immunofluorescence staining (Figure 1e).

Immunohistochemical staining of calreticulin in at least $5 \%$ of the cancer cells was observed in 227 


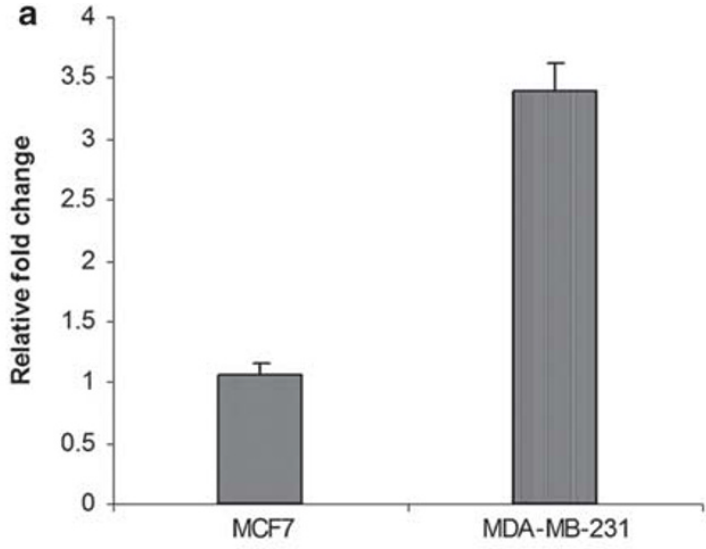

b

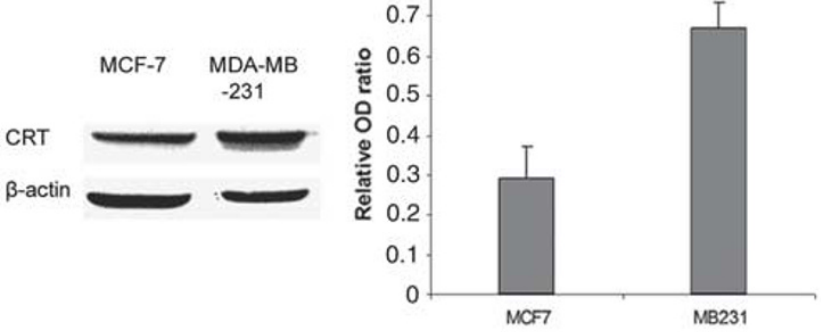

d

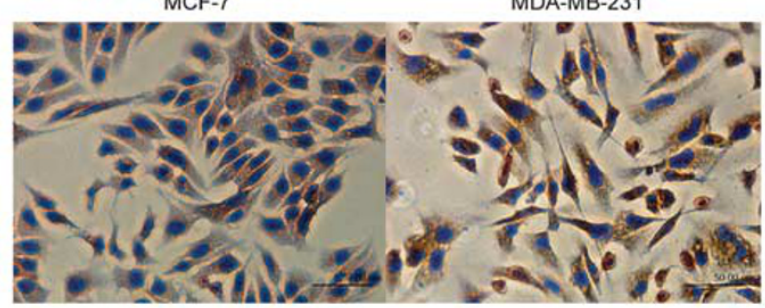

e

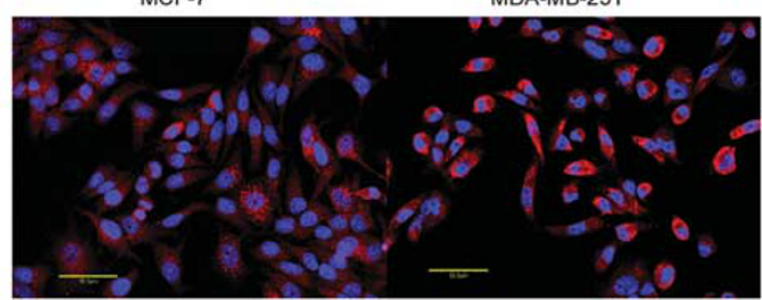

Figure 1 Expression of calreticulin in MCF-7 and MDA-MB-231 breast cancer cells. (a) Calreticulin mRNA as determined by quantitative real-time RT-PCR. MCF-12A cell line was the reference cell line. Relative expression was calculated based on $2^{-\Delta \Delta \mathrm{CT}}$. Experiments are in triplicates, Error bar=s.e.m. (b) Western blot analysis of calreticulin proteins in MCF-7 and MDA-MB-231 breast cancer cells. The housekeeping protein was $\beta$-actin. (c) Densitometric analysis of the calreticulin protein bands from the western blots. (d) Immunohistochemical staining of calreticulin protein in MCF-7 cells and MDA-MB-231 cells. Protein expression was visualized by brown DAB staining. The nucleus was stained with hematoxylin. Scale bar $=50.0 \mu \mathrm{m}$. (e) Immunofluorescence staining of calreticulin protein. The cytoplasmic localization of calreticulin was confirmed by immunofluorescent staining in both cell lines. Calreticulin is visualized by red TRIT-C staining, whereas blue DAPI stains the nucleus. Scale bar $=50.0 \mu \mathrm{m}$.

out of 228 breast cancer samples. Calreticulin immunostaining was localized to the cytoplasm of the epithelial cells with no staining observed in the stroma (Figure 2) and the percentage of positive immunostaining ranged from 5 to $100 \%$ with a mean value of $71 \%$. The intensity of calreticulin immunostaining was categorized as weak (Figure 2a), moderate (Figure 2b), and strong (Figure 2c). The negative control with absent calreticulin immunostaining is depicted in Figure 2d. Univariate regression analysis of calreticulin immunohistochemical staining in relation to the patients' clinicopathological data revealed that the weighted average intensity score of calreticulin staining was significantly and positively associated with log tumor size and development of distant metastasis (Table 2).

Every 1s.d. increase in weighted average intensity score $(\mathrm{s.d} .=0.13)$ was associated with $8.2 \%$ relative increase in tumor size and 1.6 times higher risk of developing a tumor at a distant organ later in life. On the basis that tumor size $>50 \mathrm{~mm}$ signifies advanced stage of breast cancer where chemoendocrine therapy is recommended ${ }^{26}$ and the $95 \%$ confidence interval of predicted tumor size when weighted average intensity score $=1.55$ is larger than $50 \mathrm{~mm}$ (Figure 3), the cutoff for poor prognosis was determined as 1.55. In terms of distant metastasis, the area under the ROC curve when using weighted average intensity score to predict distant metastasis (not shown) is 0.5864 (95 confidence interval: 0.47893, 0.69384). This implies that using weighted average intensity score for calreticulin immunostaining alone to predict distant metastasis does not have good predictive value. In fact, the best sensitivity and specificity is only 54.3 and $56.3 \%$, respectively, and this is achieved when using weighted average intensity score $>1.11$ to diagnose distant metastasis.

Multivariate analysis confirmed that the weighted average intensity score for calreticulin immunostaining is an independent predictor of tumor size of invasive breast ductal carcinoma and tumor recurrence (Table 3). After adjusting for patients' age, race, ER and $\mathrm{PR}$, and HER2 status, the score for calreticulin was still significantly associated with log tumor size and development of distant metastasis. There was no significant correlation of calreticulin observed with the other clinicopathological parameters. ER receptor positivity was significantly correlated to histological grade and combined histological score and HER2 positivity with histological grade.

\section{Discussion}

The association between calreticulin expression and various cancers have been previously reported by other researchers. Kageyama et al. ${ }^{27}$ observed that higher amounts of calreticulin was found in the urine samples of patients with bladder cancer and proposed calreticulin as a biomarker in bladder cancer. In gastric cancer, positive immunohistochemical staining of calreticulin was found to be 

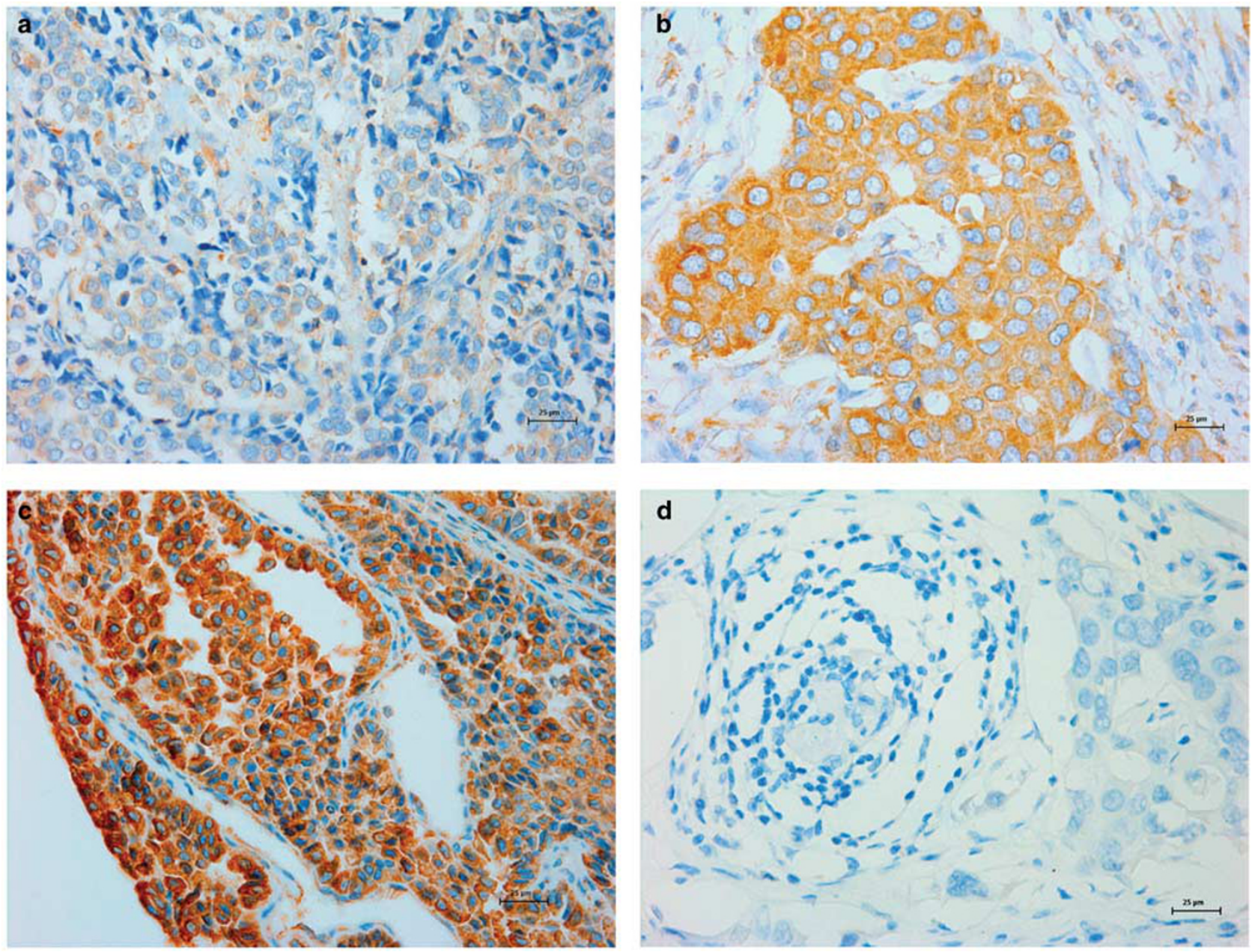

Figure 2 Immunohistochemical staining of calreticulin in breast cancer patient tissue samples. Calreticulin was specifically expressed in the cytoplasm of the epithelial cells. No staining was seen in the stroma or the lymphocytes. (a) $1+$ or weakly stained breast cancer cells. (b) $2+$ or moderately stained breast cancer cells. (c) $3+$ or strongly stained breast cancer cells. (d) Negative control. Scale bar $=25.0 \mu \mathrm{m}$.

Table 2 Univariate regression analysis for calreticulin immunostaining with clinicopathological parameters

\begin{tabular}{lrrr}
\hline & $\begin{array}{c}\text { Coefficient/ } \\
\text { odds ratio }\end{array}$ & $\begin{array}{c}\text { Standard } \\
\text { error }\end{array}$ & $\mathrm{P}$ \\
& 0.63 & 0.31 & $0.046^{*}$ \\
\hline $\begin{array}{l}\text { Log tumor size } \\
\text { Staging (1 vs 2 and 3) }\end{array}$ & 11.24 & 17.48 & 0.120 \\
$\begin{array}{l}\text { Histological grade (1 and 2 vs 3) } \\
\text { Combined histologic score }\end{array}$ & 7.58 & 8.31 & 0.064 \\
(low and medium vs high) & 13.94 & 21.44 & 0.087 \\
$\begin{array}{l}\text { Lymph node involvement } \\
\text { (yes vs no) }\end{array}$ & 0.71 & 0.74 & 0.743 \\
$\begin{array}{l}\text { Recurrence (yes vs no) } \\
\text { Metastasis (yes vs no) }\end{array}$ & 11.30 & 18.32 & 0.135 \\
& 44.56 & 71.65 & $0.017^{*}$ \\
\hline
\end{tabular}

* Statistical significance at $P<0.05$.

correlated with high microvessel density, serosal and perineural invasion, lymph node spread, and poor patient survival. ${ }^{28}$ Yet, in neuroblastoma, the most common malignancy in infants, positive immunohistochemical staining for calreticulin

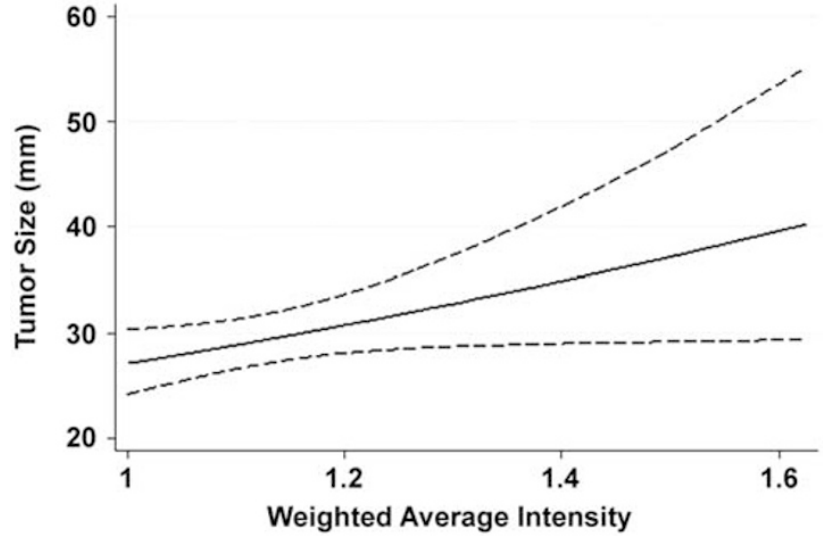

Figure 3 Predicted tumor size and its 95\% confidence intervals as a function of weighted average intensity of calreticulin.

was correlated with better prognosis and patient survival. ${ }^{23}$ Calreticulin was also found to be differentially expressed in colorectal cancer. ${ }^{29}$ Reduction 
Table 3 Multivariate analysis

\begin{tabular}{lccc}
\hline Parameters & $\begin{array}{c}\text { Odds ratio } \\
\text { (or) coefficient }\end{array}$ & $\begin{array}{c}\text { Standard } \\
\text { error }\end{array}$ & $\mathrm{P}$ \\
\hline $\begin{array}{l}\text { Log tumor size } \\
\text { Calreticulin }\end{array}$ & 0.63 & 0.31 & $0.046^{*}$ \\
$\begin{array}{l}\text { Histological grade } \\
\text { ER }\end{array}$ & 0.25 & 0.08 & $<0.001^{* *}$ \\
$\quad$ HER2 & 2.12 & 0.74 & $0.032^{* *}$ \\
$\begin{array}{l}\text { Combined histologic score } \\
\text { ER }\end{array}$ & 0.23 & 0.10 & $0.001^{* *}$ \\
$\begin{array}{l}\text { Distant metastasis } \\
\text { Calreticulin }\end{array}$ & 44.56 & 71.65 & $0.017^{*}$ \\
\hline * Statistical significance & at $P<0.05 ;$ & $* *$ statistical & significance at \\
$P<0.01$. & &
\end{tabular}

in expression of calreticulin in lung cancer cell lines was associated with an increased rate of proliferation. ${ }^{30,31}$ In a recent preliminary study of 33 breast cancer patients, Erić et $a .^{32}$ observed a potential correlation of calreticulin overexpression with axillary lymph node metastasis in breast cancer patients.

The results from our present study show that calreticulin is upregulated in the more aggressive breast cancer phenotype. We observed increased calreticulin expression in highly invasive MDA-MB231 breast cancer cells than non-invasive MCF-7 breast cancer cells at both mRNA and protein levels. As expected, calreticulin was observed to be localized in the cytoplasm of the breast cancer cells in vitro and in the tissue samples. Univariate analysis in a sample of 228 breast cancer tissues showed that calreticulin staining was significantly and positively associated with tumor size and recurrence status. Multivariate analysis confirmed a positive association between calreticulin expression level and these two parameters after controlling for possible confounders, suggesting that calreticulin is an independent predictor for tumor size and distant metastasis.

The mechanistic pathways involving calreticulin and its role in cancer remains largely unknown, although there have been extensive reports about the role of calreticulin in immunogenic cell death of cancerous cells as translocation of calreticulin together with ERp57 to the cell surface was found to be essential for death of cancer cells ${ }^{33-35}$ in response to treatment with anthracycline ${ }^{36-38}$ or exposure to UV light or $\gamma$ irradiation. ${ }^{39}$ Calreticulin has also been implicated in another pathway of cell death known as anoikis, which is a necessary cell death program for anchorage-dependent cells. ${ }^{40}$ Matricellular protein thrombospondin 1, when bound to calreticulin, induces resistance to anoikis in fibroblasts. ${ }^{41}$ Cancer cells are known to acquire enhanced survival mechanisms, which include regulating calcium homeostasis in the endoplasmic reticulum ${ }^{30}$ to counter hypoxia and endoplasmic reticulum stress. ${ }^{42}$ Calreticulin, being the main alleviator of endoplasmic stress ${ }^{43}$ could therefore have a key role in the survival of cancer cells. Calreticulin has also been reported to mediate cell adhesion and motility. ${ }^{44}$ In another study performed in kidney cancer, overexpression of calreticulin was reported to induce epithelial-mesenchymal transition in renal epithelial cells. ${ }^{45}$

In conclusion, we have observed that calreticulin protein expression is correlated with tumor size and metastatic potential in breast cancer. Further studies should be performed to elucidate the role calreticulin in breast carcinogenesis and as a potential biomarker for breast cancer.

\section{Acknowledgement}

This project was supported by the Singapore National Medical Research Council Grant NMRC/ 1081/2006 and the Singapore Cancer Syndicate Grant MS04R.

\section{Disclosure/conflict of interest}

The authors declare no conflict of interest.

\section{References}

1 Bener A, Ayub H, Kakil R, et al. Patterns of cancer incidence among the population of Qatar: a worldwide comparative study. Asian Pac J Cancer Prev 2008; 9:19-24.

2 Harding S, Rosato M, Teyhan A. Trends in cancer mortality among migrants in England and Wales, 1979-2003. Eur J Cancer 2009;45:2168-2179.

3 Meliker JR, Goovaerts P, Jacquez GM, et al. Breast and prostate cancer survival in Michigan. Cancer 2009;115:2212-2221.

4 Jemal A, Siegel R, Ward E, et al. Cancer statistics, 2009. CA-Cancer J Clin 2009;59:225-249.

5 Polyak K. On the birth of breast cancer. Biochim Biophys Acta 2001;1552:1-13.

6 Bordoni A, Probst-Hensch NM, Mazzucchelli L, et al. Assessment of breast cancer opportunistic screening by clinical-pathological indicators: a population-based study. Br J Cancer 2009;101:1925-1939.

7 Heimann R, Hellman S. Clinical progression of breast cancer malignant behavior: what to expect and when to expect it. J Clin Oncol 2000;18:591-599.

8 Sotiriou C, Pusztai L. Gene-expression signatures in breast cancer. N Engl J Med 2009;360:790-800.

9 Menard S, Casalini P, Campiglio M, et al. Role of HER2/neu in tumor progression and therapy. Cell Mol Life Sci 2004;61:2965-2978.

10 Menard S, Fortis S, Castiglioni F, et al. HER2 as a prognostic factor in breast cancer. Oncology 2001;61: 67-72.

11 Menard S, Tagliabue E, Campiglio M, et al. Role of HER2 gene overexpression in breast carcinoma. J Cell Physiol 2000;182:150-162. 
12 Qui WS, Yue L, Ding AP, et al. Co-expression of ER-beta and HER2 associated with poorer prognosis in primary breast cancer. Clin Invest Med 2009;32: E250-E260.

13 Guerra I, Algorta J, Pelayo RDD, et al. Immunohistochemical prognostic index for breast cancer in young women. Mol Pathol 2003;56:323-327.

14 Maru D, Middleton LP, Wang SJ, et al. HER-2/neu and p53 overexpression as biomarkers of breast carcinoma in women age 30 years and younger. Cancer 2005;103: 900-905.

15 Gonzalez-Angulo AM, Morales-Vasquez F, Hortobagyi GN. Overview of resistance to systemic therapy in patients with breast cancer. Adv Exp Med Biol 2007;608:1-22.

16 Gelebart P, Opas M, Michalak M. Calreticulin, a Ca2+binding chaperone of the endoplasmic reticulum. Int J Biochem Cell Biol 2005;37:260-266.

17 Michalak M, Milner RE, Burns K, et al. Calreticulin. Biochem J 1992;285:681-692.

18 Ostwald T, MacLennan D. Isolation of a high affinity calcium-binding protein from sarcoplasmic reticulum. J Biol Chem 1974;249:974-979.

19 Ellgaard L, Helenius A. Quality control in the endoplasmic reticulum. Nat Rev Mol Cell Biol 2003;4: 181-191.

20 Meldolesi J, Pozzan T. The endoplasmic reticulum Ca2+ store: a view from the lumen. Trends Biochem Sci 1998;23:10-14.

21 Michalak M, Corbett EF, Mesaeli N, et al. Calreticulin: one protein, one gene, many functions. Biochem J 1999;344:281-292.

22 Mesaeli N, Nakamura K, Zvaritch E, et al. Calreticulin is essential for cardiac development. J Cell Biol 1999;144:857-868.

23 Hsu WM, Hsieh FJ, Jeng YM, et al. Calreticulin expression in neuroblastoma-a novel independent prognostic factor. Ann Oncol 2005;16:314-321.

24 Thike AA, Chng MJ, Fook-Chong S, et al. Immunohistochemical expression of hormone receptors in invasive breast carcinoma: correlation of results of $\mathrm{H}$ score with pathological parameters. Pathology 2001; 33:21-25.

25 Koch GLE. The endoplasmic-reticulum and calcium storage. Bioessays 1990;12:527-531.

26 Goldhirsch A, Ingle JN, Gelber RD, et al. Thresholds for therapies: highlights of the St Gallen International Expert Consensus on the primary therapy of early breast cancer 2009. Ann Oncol 2009;20:1319-1329.

27 Kageyama S, Isono $\mathrm{T}$, Matsuda $\mathrm{S}$, et al. Urinary calreticulin in the diagnosis of bladder urothelial carcinoma. Int J Urol 2009;16:481-486.

28 Chen CN, Chang CC, Su TE, et al. Identification of calreticulin as a prognosis marker and angiogenic regulator in human gastric cancer. Ann Surg Oncol 2009;16:524-533.

29 Alfonso P, Nunez A, Madoz-Gurpide J, et al. Proteomic expression analysis of colorectal cancer by twodimensional differential gel electrophoresis. Proteomics 2005;5:2602-2611.
30 Bergner A, Kellner J, Tufman A, et al. Endoplasmic reticulum $\mathrm{Ca} 2+$-homeostasis is altered in small and non-small cell lung cancer cell lines. J Exp Clin Cancer Res 2009;28:25.

31 Chen CN, Su TE, Lu YC, et al. Calreticulin regulates cell proliferation and migration in gastric cancer cell line AGS. FASEB J 2007;21:928.6.

32 Eric A, Juranic Z, Milovanovic Z, et al. Effects of humoral immunity and calreticulin overexpression on postoperative course in breast cancer. Pathol Oncol Res 2009;15:89-90.

33 Clarke C, Smyth MJ. Calreticulin exposure increases cancer immunogenicity. Nat Biotechnol 2007;25: 192-193.

34 Chaput N, De Botton S, Obeid M, et al. Molecular determinants of immunogenic cell death: surface exposure of calreticulin makes the difference. J Mol Med 2007;85:1069-1076.

35 Obeid M, Tesniere A, Ghiringhelli F, et al. Calreticulin exposure dictates the immunogenicity of cancer cell death. Nat Med 2007;13:54-61.

36 Kepp O, Tesniere A, Schlemmer F, et al. Immunogenic cell death modalities and their impact on cancer treatment. Apoptosis 2009;14:364-375.

37 Kepp O, Tesniere A, Zitvogel L, et al. The immunogenicity of tumor cell death. Curr Opin Oncol 2009;21:71-76.

38 Panaretakis T, Joza N, Modjtahedi N, et al. The co-translocation of ERp57 and calreticulin determines the immunogenicity of cell death. Cell Death Differ 2008;15:1499-1509.

39 Obeid M, Panaretakis T, Joza N, et al. Calreticulin exposure is required for the immunogenicity of gamma-irradiation and UVC light-induced apoptosis. Cell Death Differ 2007;14:1848-1850.

40 Chiarugi P, Giannoni E. Anoikis: a necessary death program for anchorage-dependent cells. Biochem Pharmacol 2008;76:1352-1364.

41 Pallero MA, Elzie CA, Chen JP, et al. Thrombospondin 1 binding to calreticulin-LRP1 signals resistance to anoikis. FASEB J 2008;22:3968-3979.

42 Corazzari M, Lovat PE, Armstrong JL, et al. Targeting homeostatic mechanisms of endoplasmic reticulum stress to increase susceptibility of cancer cells to fenretinide-induced apoptosis: the role of stress proteins ERdj5 and ERp57. Br J Cancer 2007;96: 1062-1071.

43 Christensen A, Svensson K, Persson S, et al. Functional characterization of Arabidopsis calreticulin1a: a key alleviator of endoplasmic reticulum stress. Plant Cell Physiol 2008;49:912-924.

44 Coppolino MG, Woodside MJ, Demaurex $\mathrm{N}$, et al. Calreticulin is essential for integrin-mediated calcium signalling and cell adhesion. Nature 1997;386: 843-847.

45 Hayashida Y, Ihara Y, Miyata Y, et al. Overexpression of calreticulin causes epithelial-mesenchymal transition in renal epithelial cells by repressing E-cadherin gene expression: An implication to metastasis of kidney cancer. J Urol 2007;177:151-152. 